\title{
BEST APPROACH TO SECURITY IN AZURE DEVOPS
}

\section{SEREMET, Z. \& RAKIC, K.}

Abstract: Involving security in DevOps has been a challenge because traditional security methods have been unable to keep up with DevOps' agility and speed. DevSecOps is the movement that works on developing and integrating modernized security methods that can keep up with DevOps. This study is meant to give an overview of what DevSecOps is, what implementing DevSecOps means, the benefits gained from DevSecOps and the challenges an organization faces when doing so. To that end, we conducted Azure DevOps best practices, where we reviewed a selection of professional literature. We found that implementing security that can keep up with Azure DevOps is a challenge, but it can gain great benefits if done correctly.

Key words: DevOps, Cloud platform, Azure, DevSecOps, security
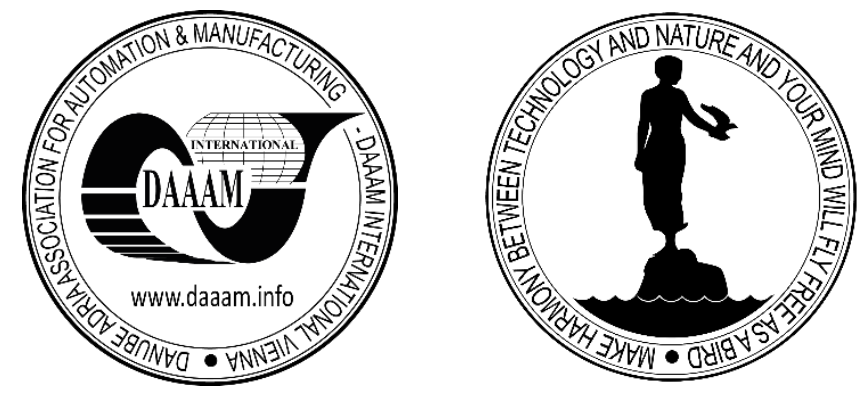

Authors' data: Asst. Prof. Dr. Sc. Seremet, Z[eljko]*; Asst. Prof. Dr. Sc. Rakic, $\mathrm{K}\left[\right.$ resimir] ${ }^{* *}, *$ University of Mostar, Trg hrvatskih velikana 1, 88000, Mostar, Bosnia and Herzegovina, zeljko.seremet@fsre.sum.ba, kresimir.rakic@fsre.sum.ba

This Publication has to be referred as: Seremet, Z[eljko] \& Rakic, K[resimir] (2021). Best Approach to Security in Azure Devops, Chapter 18 in DAAAM International Scientific Book 2021, pp.223-230, B. Katalinic (Ed.), Published by DAAAM International, ISBN 978-3-902734-31-0, ISSN 1726-9687, Vienna, Austria DOI: $10.2507 /$ daaam.scibook.2021.18 


\section{Introduction}

DevOps (Fitzgerald et al., 2017) has solved several software engineering problems, including the friction and delay in the software delivery and problem resolution. Being a manifesto designed more than a decade ago, DevOps now needs a redesign in its software development, management, and delivery, approaches. This planning can help solve the loopholes in the pipelines and cycles in DevOps. The starting principles of DevOps needed a quick response for user needs/bugs and less friction between teams - typically the development and operational teams. Although DevOps approaches these problems quite fairly, what it misses is the important aspect of modern software: the maintenance of the software.

A modern approach to software development requires the product have enough security, performance, efficiency, and a better UX to enable customers to perform their tasks. Users should also be able to know how the application uses the data it receives. One of the main emphases put on today's software is on security and data privacy. Security comes in all shapes and sizes. A solution must run on a desktop, a mobile device, a distributed environment on the cloud (Celar et al., 2011), and the smallest and lowest powered of the devices, the IoT (Internet of Things). Our software comes toe to toe with unwanted user interactions on the interface and attacks on the servers that might compromise not only the solution but also the data of other users.

So, in the second chapter, we discuss the security of DevOps (DevSecOps). The third chapter will describe one of the more influential DevOps cloud platforms (Microsoft Azure DevOps), and the fourth chapter the security aspect with Azure policies. Finally, in the fifth chapter, Azure Security Best Practices are proposed, and in the sixth, a conclusion is given.

\section{DevSecOps process}

Introduction of agile development methodology was a huge leap towards more organized and ideal development cycle. But where agile tends to solve the communication problems with developer and the client DevOps strives to also solve similar problem between developer and operations. Therefore, many view DevOps as the logical continuation of agile method. This can also be noticed when looking into DevOps culture since DevOps shares multiple similarities with agile development. DevOps culture got introduced because it was noticed that the developers and IT operators rarely worked together as a team toward the same goal, but they worked as separate units. (Myrbakken et al., 2017)

With everyone moving to the cloud, there's been so much hype over DevOps and how it can make processes faster, easier, and more efficient. As someone in security though, he feels fear that there is no emphasis on moving to the cloud securely. Enter DevSecOps. (Clarke et al., 2017)

DevSecOps is about introducing security earlier in the software development life cycle (SDLC) (Fig. 1). The goal is to develop more secure applications by making everyone involved in the SDLC responsible for security. 
Having business, tech, and security work together to produce secure products seems like a dream come true. Maybe too good to be true? Let's investigate more and see if DevSecOps can be the silver bullet we all need in building secure products.

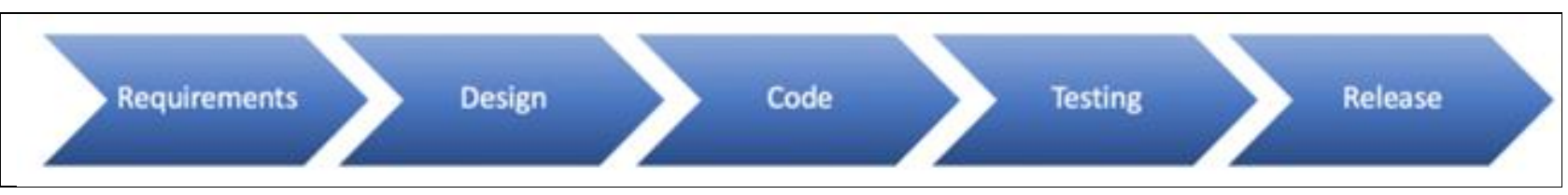

Fig. 1. DevSecOps Life Cycle

First, let's talk about why we need DevSecOps. Years ago, software products followed the waterfall methodology, a linear sequential approach for developing a product that concluded with a "big-bang" release. (Chatterjee, 2020)

With the shift to cloud computing and dynamic provisioning of resources, developers have gained numerous benefits around speed, scale, and cost of application development. These benefits lend themselves so nicely to the adoption of the DevOps movement. DevOps strongly advocates for automation and monitoring at all steps of the SDLC.

The goal is for shorter development cycles, increased deployment frequency and more dependable releases, all aligned with business objectives. But stable infrastructure and applications does not equal secure infrastructure and applications. In the waterfall lifecycle, security checks were put at the end before the product was released. Security was seen as a roadblock, the last gate-check, on the way to a production release. Many things have changed regarding how applications are developed, but not how security is viewed. (Ahmad Zeeshan, A., 2020)

The most teams use the agile methodology for software development. In an agile environment, the focus is on rapid delivery. By using iterative planning and feedback results, teams can continuously align product deliverables to business needs. The adaptability to changing requirements is great for delivering a meaningful product, but if you're releasing a new version of your product every week, when do you test for security vulnerabilities? Unfortunately, traditional security processes have not kept pace in agile/DevOps environments rendering security to become a major roadblock in software development where it is usually bypassed.

If it's not bypassed, the development team rarely has enough time to address all the issues before the product goes live which means that an insecure application lives somewhere on the internet. The ironic part is that ignoring security to avoid the risk of missing a deadline puts more risk into the application. Security defects in the SDLC can lead to serious vulnerabilities like a breach caused by bad code. Therefore, we need DevSecOps.

In DevSecOps the two seemingly opposing goals, "speed of delivery" and "secure code", are merged into one streamlined/automated process. The intent of DevSecOps is to build on the mindset that "everyone is responsible for security". It is about pushing security left and automating core security tasks. 
We can push security to the left of the SDLC to ensure that application security starts as one codes. By shifting left, teams can quickly discover and analyse vulnerabilities and then adapt their code to mitigate against those vulnerabilities. DevSecOps allows developers to focus on writing high quality and secure code, enabling teams to release titanium applications.

The benefits are simple:

- security from the start minimizes the chance of vulnerabilities

- having automated security tools running in pipelines is that it lets security team members focus on the high-hanging fruit

- better collaboration and communication between dev and security teams

- improved operational efficiencies across security and the enterprise

However, there are challenges associated with DevSecOps. Even with security baked into a pipeline, there are still ways to circumvent security checkpoints. Let's take an example where a vulnerability scanner is being used to block a build/deployment if a certain vulnerability is found. As a developer, you know what that vulnerability is and you know your code has it, but you really need to do this release. So, you find a way to hide pieces of the code that you know will fail a security scan, resulting in a successful build. Another common situation is when teams decide to break their build if there's a presence of one or more findings of a certain severity. For example, a team may say that they do not want to break their build unless the finding is high or critical. While this certainly helps identify and address high priority issues immediately, the consequence is that medium and low findings make it to production builds.

Now that we've gone over the pros and cons of DevSecOps, how does one implement it? The traditional DevOps tools such as Jenkins and Git are a must have to build the foundation of your DevOps pipeline. There are many security tools in the marketplace ranging from open source to proprietary solutions. Many of these can be integrated into your existing pipelines.

Below is a popular list security tool:

- Checkmarx: A SAST (Static Application Security Testing) Tool that analyzes an application's code for flaws which are indicative of security vulnerabilities.

- WhiteSource: An open-source vulnerability scanner, which runs automatically and continuously in the background, tracking the security, licensing, and quality of open-source components and matching them against WhiteSource's comprehensive database of open-source repositories.

- Zaproxy: The OWASP Zed Attack Proxy (ZAP) is an easy-to-use integrated penetration testing tool for finding vulnerabilities in web applications.

So, is DevSecOps the magical cure to all our problems? Maybe not all our problems, but for many it is irreplaceable, and this is one hype train that should be ready to board and go to the end. (Chatterjee, 2020) 


\section{DevOps cloud platforms}

App creators have many DevOps tools to choose from, and the sheer number is enough to make anyone's head spin. One of the better available DevOps tools, Microsoft Azure DevOps, will be described in more detail below.

\subsection{Microsoft Azure DevOps}

Microsoft Azure DevOps is the Microsoft's take on DevOps solutions. This solution is the evolved form of the former Visual Studio Team Services.

Azure DevOps includes five different services:

- Azure Boards: This covers agile planning, work item tracking, and visualization, and reporting tools.

- Azure Repos: This offers a cloud-hosted private Git repository, with pull requests, advanced file management, and other benefits.

- Azure Pipelines: This is a language, platform, and cloud-agnostic CI/CD (Continuous Integration/Continuous Delivery) platform with support for containers or Kubernetes.

- Azure Test Plans: This service provides an integrated, all-in-one planned, and exploratory testing solution.

- Azure Artifacts: The artifacts in question provide developers with integrated package management, including support for Maven, npm, Python, and NuGet package feeds from either public or private sources.

These services are extensible and flexible, and they can be used with different platforms and clouds so the user may choose not to use the default Azure cloud solution when using these Azure tools. Azure Repos, Test Plans and Artifacts can be integrated with Azure Pipeline's CI/CD. Azure DevOps also offers a variety of extensions and support for user created extensions. There are extensions for example for Docker, Slack, GitHub, Sonar Qube and AWS tools. Many of these extensions are offered free, but some may require paid subscription. (Microsoft Corporation, 2020)

Azure DevOps Services' pricing model will mostly depend on how big of a team is working with Azure Cloud. Even with the free version users gain access to Azure Boards, Azure Repos, Azure Artifacts, Azure Test Plans and Azure Pipelines. These $\mathrm{CI} / \mathrm{CD}$ pipelines costs change how much traffic and how many multiple parallel jobs can be done at the same time. Azure Portal is also needed to host virtual machines when deploying the apps. Costs for Azure Portal can be changed with different subscriptions.

\subsection{Workflow in Azure}

Workflow with Azure is quite simple, but there are few things to be noted. First user creates a branch inside Azure DevOps and then either clones the Azure repository if this is the first time working with project, or, if user already has repository set in the local environment, they can just pull the new branch and start working on it. 
After the changes are made and user is ready to push the changes to repository, a commit is needed to be made that describes the changes. After the commit, the user can push changes to Azure DevOps. Then from inside the Azure DevOps site, when clicking on the "Repos" tab there is notification that states there are new changes in the branch. It also proposes making a pull request out of it. (Priyadarsini et al., 2020)

Pull requests are used by development team to review the changes to the code. The changes can be approved, rejected and other team members can comment on changes and suggest alternative fixes for the code. Pull requests can be managed from under the "Repos" tab and selecting "Pull requests". Here, users can create a new pull request for the branch they have been working on. If someone is set as a reviewer for code changes made by others, they will see those requests here. When the right number of reviewers have accepted the changes the pull request can be created. This will merge the changed branch to a master branch. (Microsoft Corporation, 2020)

Next is the continuous pipeline's turn to start doing its part. This pipeline can be modified to automatically start the deployment process when the master branch is changed. This means that it builds the code and does the tests included in it. There is also an artifact created after the build process. The artifact is then deployed to the virtual environment by the deploy stage. After the pipeline has run its stages, the changes can be seen in the deployment environment. (Microsoft Corporation, 2020)

\section{Security with Azure Policies}

Azure Policies are as important as the cloud platform itself and very relevant in DevOps. It is not just a security tool but to enforce organization structure and compliance. It can be used to manage costs, ensure security best practices, and reduce the scope of the search when things go wrong and help in default and custom policies. In DevOps, Azure Policies are vital for the providing technical security and consistency in enforcing industry and business standards then ultimately compliance.

Some key points are shown as follows:

- They give us a way to manage costs by restricting specific resources.

- Because of the policy-enabled secure architecture, we can determine the scope, source, and solution of problems more quickly.

- Through policy-enabled configuration management, unexpected or even unwanted configuration changes can be prevented. (Microsoft Corporation, 2020)

\subsection{Custom Policy Definitions}

Azure policies are written in JSON (JS Object Notation) and can be deployed in the portal or the CLI.

Policies have two modes:

- Indexed: This evaluates only resource types that support tags and locations.

- All: This evaluates resource groups, subscriptions, and all resource types. 
However, the rules themselves follow a simple if-then structure, where if the conditions in the policy rule are met, the effect specified in the "then" block occurs. (Microsoft Corporation, 2020)

\subsection{Network Security Groups}

Network Security Groups (NSGs) are a collection of networking rules that dictate the flow of traffic to and from resources in Azure. When NSGs are created, there are numerous customization that can be made and some of them are:

- Name

- Priority: Ranges from 100-4096

- Source or Destination: A single IP or CIDR can be considered

- Protocol: All the networking protocols

- Direction: Tells us whether the rule applies to the traffic into the network

- Port Range: 3000-30010

- Allow or Deny Action = Allow (Choudary, 2017)

\section{Azure Security Best Practices}

Azure DevOps with a comprehensive list of best practices while considering security include:

- Treat Identity as security perimeter (like for verification and access. Identity management must be centralized).

- It is not necessary to have two references to an identity.

- Enable single sign-on so users can use the same credentials to resources so they can easily control things.

- There must be strong network controls to connect VM across networks and control the traffic for the VMs.

- Segment subnets must be controlled logically while resources belong to the strongest security zone.

- More network space needs to be allocated than is required when setting up the infrastructure.

- Don't assign NSG rules with broad ranges and be particular with who has access to what in the system.

- Finally, it is necessary to explore the goodies of the Azure security centre. (Microsoft Corporation, 2021)

\section{Conclusion}

This paper presents the research we did on DevSecOps to find out how DevSecOps can be defined, what doing DevSecOps means for an organization in regard to what principles and practices they should adhere to, what challenges they would face attempting to adopt DevSecOps, the benefits if it's done successfully and how it has evolved from the need to implement security in DevOps to what could seem like a movement on its own. 
We have identified several challenges and benefits for implementing the security of one of the major cloud platforms, Microsoft Azure DevOps. The challenges we identified should not be seen as deterrents to implementing DevSecOps, but a symptom of its youth. As DevSecOps matures, better methods, practices, tools etc. can probably overcome them. The benefits we identified indicates it is maturing, by for example resulting in less unplanned work and a decrease in manual labour.

As future work, it would be interesting to conduct surveys on organizations to possibly expand this study's coverage on DevSecOps. It is also of interest to investigate this study's suggested practices: observing practices effects on the surrounding environments (development, operations, business, customers) confirm the proposed best practices. A possibility would then be to investigate and propose possible architectures or frameworks for implementing DevSecOps, for example look at continuous software engineering while using a microservices architecture.

\section{References}

Ahmad Zeeshan, A. (2020). DevSecOps for .NET Core, ISBN: 978-1-4842-5850-7, Apress, Berkeley, CA

Celar, S.; Seremet, Z. \& Turic, M. (2011). Cloud computing: definition, characteristics, services and models, Annals of DAAAM for 2011 \& Proceedings of the 22nd International DAAAM Symposium / Katalinic, Branko - Vienna: DAAAM International Vienna, 2011, 0001-0002

Choudary, A. (2017). Secure Your Applications Using Azure Virtual Network Available from: https://medium.com/edureka/azure-virtual-network-securing-yourapplications-using-vpc-744eba3aa5b1 Accessed: 2021-10-19

Clarke, P. M., O'Connor, R.V., Elger, P. (2017). Continuous software engineering-a microservices architecture perspective. J. Softw. Evol. Proc. 2017, e1866 devops/user-guide/services?view=azure-devops Accessed: 2020-05-19.

Fitzgerald, B. \& Stol, K. J. (2017). Continuous software engineering: a roadmap and agenda, J. Syst. Softw. 123, 176-189

K. Priyadarsini, K.; Fantin Irudaya Raj, E.; Yasmine Begum, A. \& Shanmugasundaram, V. (2020). Comparing DevOps procedures from the context of a systems engineer, Materials Today: Proceedings, Volume 47, Part 19

Microsoft Corporation. Azure Identity Management and access control security best practices Available from: https://docs.microsoft.com/enus/azure/security/fundamentals/identity-management-best-practices Accessed: 202111-19

Microsoft Corporation: What features and services do I get with Azure DevOps? Available from: https://docs.microsoft.com/en-us/azure/

Myrbakken H. \& Colomo-Palacios R. (2017). DevSecOps: A Multivocal Literature Review. In: Mas A., Mesquida A., O'Connor R., Rout T., Dorling A. (eds) Software Process Improvement and Capability Determination. SPICE 2017. Communications in Computer and Information Science, vol 770. Springer, Cham.

R. Chatterjee (2021). Introduction to IT Security. In: Red Hat and IT Security. Apress, Berkeley, CA. 